\title{
Trials of Novel Oral Anticoagulants for Stroke Prevention In Patients with Non-valvular Atrial Fibrillation
}

\author{
Jonathan L. Halperin ${ }^{\mathrm{a} *}$ and Paul Dorian ${ }^{\mathrm{b}}$ \\ ${ }^{a}$ The Cardiovascular Institute, Mount Sinai Medical Center, New York, NY USA; ${ }^{b}$ Division of Cardiology, University of \\ Toronto, St Michael's Hospital, Toronto, ON Canada
}

\begin{abstract}
Patients with non-valvular atrial fibrillation (AF) face an increased risk of stroke compared with those in normal sinus rhythm. The vitamin $\mathrm{K}$ antagonist warfarin, available for over half a century, is highly effective in reducing the risk of stroke in patients with AF, but it is a difficult drug to use properly. As a result, it is challenging to keep the anticoagulant effect of warfarin in the desired range. Newer oral anticoagulants (NOACs) that directly inhibit Factor IIa (thrombin) or Factor Xa provide reliable anticoagulation when administer in fixed oral doses without routine coagulation monitoring. This manuscript will review in detail the pivotal trials of these NOACs that led to their approval as well as comment on the factors that should influence their selection.
\end{abstract}

Keywords: Anticoagulation, antithrombotic therapy, atrial fibrillation, factor IIa inhibitors, factor Xa inhibitors, stroke, thrombin, thromboembolism.

\section{INTRODUCTION}

Patients with non-valvular atrial fibrillation $(\mathrm{AF})$ face an increased risk of stroke compared with those in normal sinus rhythm, though the magnitude of risk varies in relation to age and other clinical features [1,2]. The vitamin $\mathrm{K}$ antagonist warfarin, available for over half a century, is highly effective in reducing the risk of stroke in patients with AF [3], but its use is complicated by variable dose-response due to genetic factors and multiple food and drug interactions, resulting in the need for frequent monitoring of anticoagulation intensity and frequent dose adjustments. As a result, it is challenging to keep the anticoagulant effect of warfarin in the desired range; the INR is in the optimum range of 2.0-3.0 about $50 \%$ of the time in most clinical settings. Newer oral anticoagulants (NOACs) that directly inhibit Factor IIa (thrombin) or Factor Xa provide reliable anticoagulation when administered in fixed oral doses without routine coagulation monitoring.

\section{DIRECT THROMBIN INHIBITORS}

Evaluation of the oral, direct thrombin inhibitor ximelagatran in the SPORTIF III and V trials [4] found it efficacious, but it was withdrawn from further development because of hepatotoxicity. Dabigatran, another oral Factor IIa inhibitor, was compared with warfarin in the RE-LY trial of 18,113 patients with AF at risk for stroke; patients were randomly assigned to one of two blinded doses of dabigatran etexilate, $110 \mathrm{mg}$ or $150 \mathrm{mg}$ twice daily, or open-label, doseadjusted warfarin (target INR 2.0-3.0) [5, 6]. The mean $\mathrm{CHADS}_{2}$ score of enrolled patients was 2.1, and the median duration of follow up was 2.0 years. The primary efficacy

*Address correspondence to this author at the Cardiovascular Institute, Mount Sinai Medical Center, Fifth Avenue at $100^{\text {th }}$ Street, New York, NY 10029-6574, USA; Tel: (212) 241-7243; Fax: (212) 831-2195; E-mail: jonathan.halperin@mssm.edu outcome was the rate of all stroke (ischemic or hemorrhagic) or systemic embolism. This occurred at rates of $1.71 \% /$ year in the group assigned to warfarin, $1.54 \% /$ year in those randomized to dabigatran, $110 \mathrm{mg}$ b.i.d. $(\mathrm{p}<0.001$ for noninferiority to warfarin), and $1.11 \%$ /year with dabigatran, $150 \mathrm{mg}$ b.i.d. $(\mathrm{p}<0.001$ for superiority to warfarin). Both doses of dabigatran were associated with significantly lower rates of hemorrhagic stroke (intracerebral hemorrhage) compared to warfarin. The higher dose yielded a rate of major bleeding similar to that with warfarin but a higher rate of gastrointestinal bleeding. All-cause mortality was lower with dabigatran (4.13\%/year for warfarin, $3.75 \% / y e a r$ for dabigatran, $110 \mathrm{mg}$ bid, 3.64\%/year for dabigatran, $150 \mathrm{mg}$ bid), but did not reach statistical significance $(\mathrm{p}=0.13$ and $\mathrm{p}=0.051$ for the 110 $\mathrm{mg}$ and $150 \mathrm{mg}$ bid doses, respectively). These findings led to regulatory approval of dabigatran etexilate at the $150 \mathrm{mg}$ twice daily dose to reduce the risk of stroke and systemic embolism in patients with nonvalvular AF in the U.S., Canada, Europe and many other countries as the first novel anticoagulant for this indication. Unlike regulators in most other countries, the U.S Food and Drug Administration did not approve the lower $110 \mathrm{mg}$ b.i.d. dose formulation. Although patients with severe renal impairment (creatinine clearance $\left[\mathrm{Cr}_{\mathrm{Cl}}\right] \leq 30 \mathrm{~mL} / \mathrm{min}$ ) were excluded from RE-LY, the FDA approved an even lower dose of $75 \mathrm{mg}$ twice daily for patients with $\mathrm{Cr}_{\mathrm{Cl}} 15-30 \mathrm{~mL} / \mathrm{min}$, based on pharmacokinetic modeling, despite a lack of clinical data [7]. Dabigatran has been approved in Canada and Europe for prevention of venous thromboembolism (VTE) in patients undergoing total hip or knee replacement surgery and in the U.S. for treatment of patients with VTE and prevention of recurrence.

\section{DIRECT FACTOR XA INHIBITORS}

In the ROCKET AF trial, the oral direct Factor Xa inhibitor, rivaroxaban, proved noninferior to warfarin for preven- 
tion of stroke or systemic embolism and resulted in significantly fewer fatal bleeding events and hemorrhagic stroke [8]. The trial included 14, 264 patients with AF at generally higher risk of stroke than those enrolled in the RE-LY study (mean $\mathrm{CHADS}_{2}$ score $=3.5$ ). More than half of the enrolled patients $(55 \%)$ had sustained previous stroke, systemic embolism or transient ischemic attack (TIA). Patients were randomized to receive dose-adjusted warfarin (goal INR 2.0$3.0)$ or fixed-dose rivaroxaban, $20 \mathrm{mg}$ once daily $(15 \mathrm{mg}$ daily for those with $\mathrm{Cr}_{\mathrm{Cl}} 30-49 \mathrm{ml} / \mathrm{min}$ ) double-blind. Over a median follow-up period of 707 days, the primary efficacy endpoint was reached in 188 patients with rivaroxaban group and in 241 with warfarin group (1.7 and 2.2 per 100 patientyears, respectively; $\mathrm{p}<0.001$ for noninferiority) in the perprotocol on-treatment population. When analyzed by intention-to-treat (ITT), the primary event rates were 2.1 per 100 patient-years in the warfarin group and 2.4 per 100 patientyears in the rivaroxaban group, meeting the criterion for noninferiority $(p<0.001)$ but not superiority $(p=0.12)$. There was an accumulation of events when those randomized to rivaroxaban were transitioned to open-label warfarin at the end of the study treatment period and anticoagulation intensity may have been below the therapeutic range for nearly two weeks.

There was no difference in the rate of major and clinically relevant non-major bleeding (14.5 vs. 14.9 per 100 patient-years in the warfarin and rivaroxaban groups, respectively; $\mathrm{p}=0.44$ ). Patients randomized to rivaroxaban experienced more gastrointestinal bleeding, more often developed declines in hemoglobin concentration of $2 \mathrm{~g} / \mathrm{dL}$ and more often required blood transfusion than those in the warfarin group, but there were significantly lower rates of hemorrhagic stroke $(0.5$ vs 0.7 per 100 patient-years, $\mathrm{p}=0.02)$ and fatal bleeding $(0.2$ vs $0.5 \%, \mathrm{p}=0.003)$ with rivaroxaban compared with warfarin. The difference in allcause mortality (1.9 and 2.2 per 100 patient-years, $p=0.073$ ) with rivaroxaban compared with warfarin, respectively, did not satisfy criteria for statistical significance. Rivaroxaban has been approved by the FDA, Canadian and European health authorities to reduce the risk of stroke and systemic embolism in patients with non-valvular AF and in different dosing regimens for prevention of VTE in patients undergoing major orthopedic surgery of the hip or knee and for treatment of deep venous thrombosis and/or pulmonary embolism.

Another Factor Xa inhibitor, apixaban, was evaluated in the ARISTOTLE trial [9], in which 18,201 patients with non-valvular $\mathrm{AF}$ and at least one additional risk factor for stroke (mean $\mathrm{CHADS}_{2}$ score 2.1) were randomized to apixaban, $5 \mathrm{mg}$ b.i.d. (2.5 $\mathrm{mg}$ b.i.d. for those with two or more of the following factors associated with increased drug exposure: age $\geq 80$ years, body weight $\leq 60 \mathrm{~kg}$, or serum creatinine $\geq 1.5 \mathrm{mg} / \mathrm{dL}$ ) or dose-adjusted warfarin (target INR 2.0-3.0). A total of $19 \%$ of patients had a previous TIA, stroke, or systemic embolism. The mean follow-up was 1.8 years. The primary efficacy outcome (stroke or systemic embolism) occurred less often in the apixaban group (1.27\%/year) than in the warfarin group $(1.60 \% / y e a r ; p<0.001$ for noninferiority; $\mathrm{p}=0.01$ for superiority). The apixaban group also exhibited lower rates of hemorrhagic stroke ( 0.24 vs. $0.47 \%$ /year; $\mathrm{p}=<0.001)$, major bleeding (2.13 vs. $3.09 \% /$ year; $\mathrm{p}<0.001)$, intracranial hemorrhage ( 0.33 vs. $0.80 \%$ per year; $\mathrm{p}<0.001)$ and all-cause mortality (3.52 vs. 3.94\%/year; $\mathrm{p}=0.047)$. There were small and statistically insignificant reductions in rates of major gastrointestinal bleeding, myocardial infarction (MI) and death from cardiovascular causes.

A separate large clinical trial, AVERROES, compared apixaban to aspirin for prevention of stroke and systemic embolism in 5,599 patients with AF for whom VKA therapy was deemed unsuitable [10]. The trial was terminated prematurely when apixaban showed convincingly superior efficacy in reducing the risk of stroke or systemic embolism without increases in the risk of major bleeding or hemorrhagic stroke. Based on the results of the ARISTOTLE and AVERROES trials, apixaban has been approved in the U.S. and in Europe for prevention of VTE in patients undergoing major orthopedic surgery of the hip or knee and for stroke prevention in patients with AF in Europe, Canada and the U.S.

Two doses of the direct Factor Xa inhibitor, edoxaban, 30 and $60 \mathrm{mg}$ once daily, in comparison to warfarin for prevention of stroke in patients with AF were investigated in the ENGAGE AF-TIMI 48 trial of 21,105 patients [11]. Results were reported in 2013, and the drug is currently undergoing regulatory review.

\section{CRITICAL ASSESSMENT AND COMPARISON OF THE TRIAL RESULTS}

Assessment of the completed trials comparing the FDAapproved new oral anticoagulants ("NOACs") with warfarin reveals several similarities and differences (Fig. 1). Dabigatran, rivaroxaban and apixaban are all noninferior to warfarin for prevention of stroke and systemic embolism in patients with $\mathrm{AF}$, and each of these agents significantly reduced the rate of hemorrhagic stroke. In an ITT analysis, dabigatran $150 \mathrm{mg}$ b.i.d. and apixaban significantly reduced the rate of all cause stroke. Only dabigatran, $150 \mathrm{mg}$ b.i.d. was statistically superior to warfarin for prevention of ischemic stroke. All were noninferior to warfarin with respect to avoidance of major bleeding, and apixaban and dabigatran, $110 \mathrm{mg}$ b.i.d., reduced major bleeding. All 3 drugs led to a $9-12 \%$ reduction in all-cause mortality, although this finding was significant only for apixaban. All trials reported reductions in fatal or life-threatening bleeding compared with warfarin. Rivaroxaban and dabigatran, $150 \mathrm{mg}$ b.i.d., increased gastrointestinal hemorrhage but this was not observed with apixaban or the lower dose of dabigatran, $110 \mathrm{mg}$ b.i.d. Since trials directly comparing one NOAC with another have not been conducted, and there are important differences in patient populations, study designs and methodological factors, we cannot draw conclusions about the relative safety or efficacy of the individual drugs or classes (Factor IIa and Factor Xa inhibitors) based on data currently available. The lack of comparative effectiveness data creates a challenge to practitioners, but the collective data make it clear that, considered as a group with respect to safety and efficacy, the NOACs offer favorable net clinical benefit compared to warfarin for many patients with AF.

\section{MYOCARDIAL INFARCTION}

There was no significant difference in rates of MI between the NOACs and warfarin in the contemporary trials. 

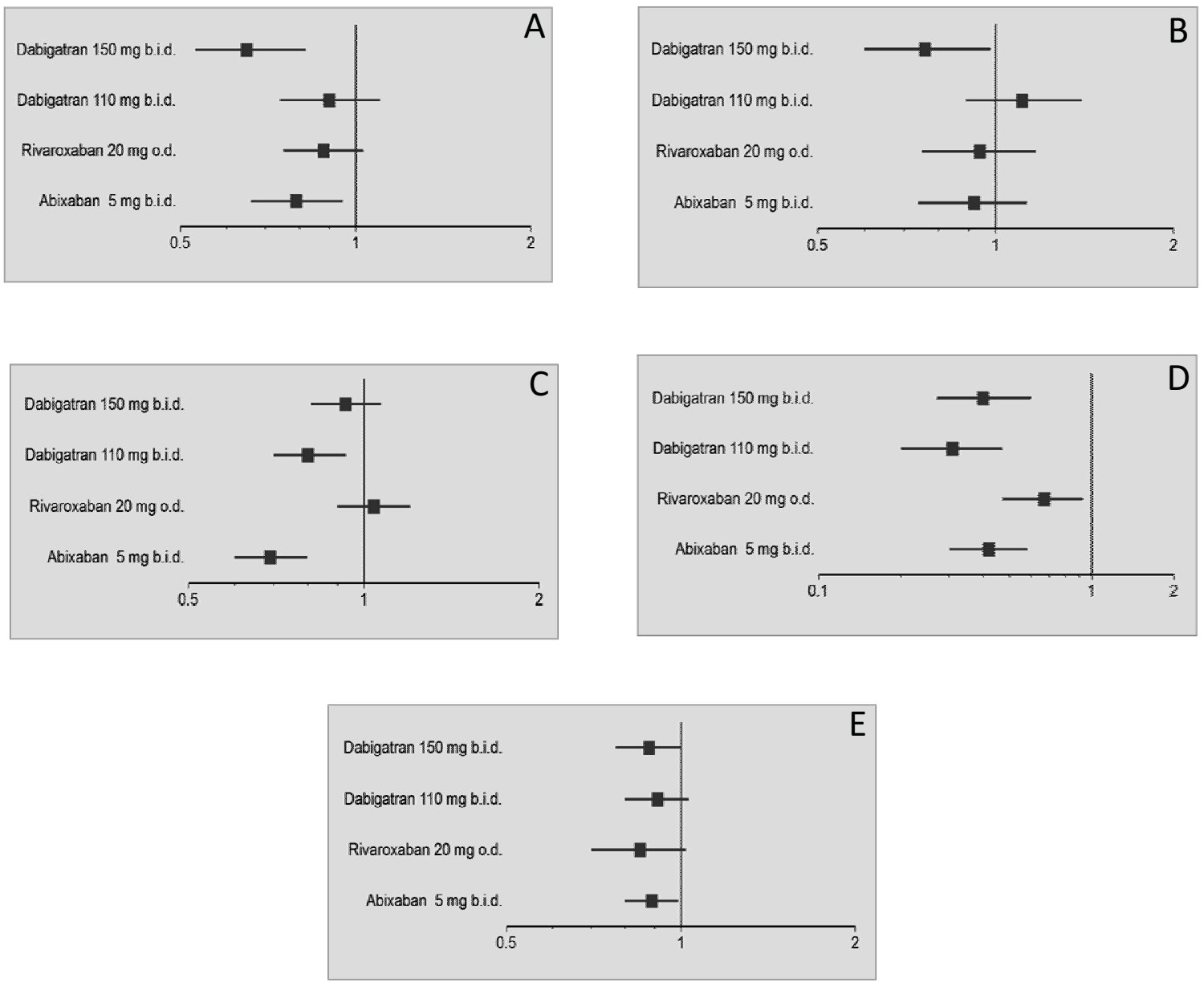

Fig. (1). Relative rates of (A) all stroke and systemic embolism, (B) ischemic stroke. (C) major bleeding, (D) hemorrhagic stroke, and (E) all-cause mortality compared to warfarin in the RE-LY trial of dabigatran [6], ROCKET-AF trial of rivaroxaban [8] and ARISTOTLE trial of apixaban [9].

In trials of dabigatran, however, there was a trend - not statistically significant - of higher rates of MI than observed in patients treated with warfarin [6, 12]. Metaanalysis of seven randomized trials suggested that dabigatran might be associated with an increased risk of MI, but the bulk of the experience was derived from the RE-LY trial. In most studies on patients with AF, rates of MI are considerably lower than those of stroke, and the risk of subsequent MI was not increased in those with coronary disease of prior MI treated with dabigatran relative to warfarin in RE-LY or in a subsequent analysis of postmarketing experience among Medicare enrollees in the U.S. conducted by the FDA $[6,12,12 \mathrm{a}]$.

No dosing recommendations can be made based on currently available evidence for use of the NOACs in patients with AF who are receiving dual antiplatelet therapy (DAPT) following acute coronary syndrome (ACS) [13-15] or percutaneous coronary intervention (PCI). Anticoagulated patients treated concurrently with platelet inhibitor drugs face a significantly higher risk of bleeding than those receiving either type of antithrombotic agent alone, and among anticoagulated patients with AF in the RE-LY trial, antiplatelet therapy was the most important independent, modifiable risk factor for hemorrhagic stroke [16].

\section{SECONDARY PREVENTION}

Patients with AF who have suffered previous stroke or TIA face a risk of recurrent stroke approximately 2.5 times greater than those without prior events [17] and are also at greater risk of bleeding [18]. In the subgroup of 3,623 patients with previous stroke or TIA randomized in the RE-LY trial, rates of stroke or systemic embolism were $2.78 \% /$ year in the warfarin group, compared with $2.32 \%$ /year with dabigatran, $110 \mathrm{mg}$ b.i.d. (relative risk [RR] 0.84, 95\% confidence interval [CI] $0.58-1.20$ ), and $2.07 \%$ /year) with dabigatran, $150 \mathrm{mg}$ b.i.d. (RR $0.75 ; 95 \%$ CI $0.52-1.08$ ). The rate of major bleeding was significantly lower in patients with dabigatran, $110 \mathrm{mg}$ b.i.d. (RR 0.66, 95\% CI 0.48-0.90) but similar in patients randomized to $150 \mathrm{mg}$ b.i.d. (RR 1.01; 95\% CI $0.77-1.34$ ) compared to warfarin. The effects of dabigatran in patients with previous stroke or TIA and did not differ from those in patients without prior thromboembolic events for any of the outcomes in the RE-LY trial other than vascular death, which was lower in the $110 \mathrm{mg}$ b.i.d. group compared with warfarin group (interaction $\mathrm{p}=0.038$ ) [19].

In the ARISTOTLE and RE-LY trials, 19 and 20\%, respectively, had experienced previous stroke or TIA. In the ROCKET AF trial, 52 percent of enrolled patients had prior cerebral ischemic events (excluding systemic embolism out- 
side the central nervous system). While the absolute event rates were generally higher in those with prior stroke or TIA, there was no significant difference in relative efficacy or safety of rivaroxaban compared with warfarin in this population compared with those in the entire ROCKET AF trial population. The event rate for the primary endpoint with rivaroxaban ( 2.79 per 100 patient-years) compared with warfarin (2.96 per 100 patient-years) was consistent in patients with previous stroke or TIA (hazard ratio [HR] 0.94, 95\% CI $0.77-1.16)$ and those without (1.44 vs $1.88 \% / 100$ patientyears; HR 0.77, 95\% 0.58-1.01; interaction $\mathrm{p}=0.08$ ) [20]. Rates of major and non-major clinically relevant bleeding per 100 person-years were also consistent among patients with previous stroke or TIA (13.31/100 patient-years on rivaroxaban vs 13.87/100 patient-years on warfarin; HR 0.96, 95\% CI $0.87-1.07)$ and those without (16.69 vs $15.19 / 100$ patientyears; HR 1.10, 95\% CI 0.99-1.21; interaction $\mathrm{p}=0.08)$.

In the secondary prevention subgroup of the ARISTOTLE trial, the rate of stroke or systemic embolism was $2.46 / 100$ patient-years in the apixaban group and 3.24 in the warfarin group (HR 0.76, 95\% CI 0.56-1.03); in those without previous stroke or TIA, the rate of stroke or systemic embolism was 1.01/100 patient-years with apixaban and 1.23 with warfarin (HR $0.82,95 \%$ CI $0.65-1.03$; interaction $\mathrm{p}=0.71)$. The absolute reduction in the rate of stroke and systemic embolism with apixaban $v s$ warfarin was $0.77 / 100$ patient-years (95\% CI -0.08-1.63) in patients with and 0.22 $(-0.03$ to 0.47$)$ in those without previous stroke or TIA. The absolute reduction in major bleeding with apixaban compared with warfarin was $1.07 / 100$ patient-years $(95 \%$ CI $0.09-2.04)$ in patients with and $0.93(0.54-1.32)$ in those without previous stroke or TIA [21].

Individually, each of these subgroup analyses was underpowered to demonstrate with statistical confidence the noninferiority or superiority of the NOACs compared to warfarin for secondary prevention of ischemic events in patients with AF who had experienced prior stroke or TIA, nor can conclusions be drawn about the performance of one of the novel agents vs. another. Meta-analysis of the 14, 527 patients with prior stroke or TIA randomized in the three pivotal trials found the NOACs associated with a significant reduction of stroke and systemic embolism (odds ratios [OR] $0.85,95 \%$ CI 074-0.99]; relative RR 14\%; absolute RR, 0.7\%; number needed-to-treat [NNT], 134 over 1.8-2.0 years) compared with warfarin. The NOACs were also associated with a significant reduction in major bleeding compared with warfarin (OR 0.86, 95\% CI 075-0.99; relative RR $13 \%$; absolute RR $0.8 \%$; NNT 125), driven mainly by the significant reduction of hemorrhagic stroke (OR 0.44, 95\% CI 032-0.62; relative RR 57.9\%; absolute RR 0.7\%; NNT 139). Hence, preservation of their relative efficacy and safety and conformity with the overall trial results supports the use of the NOACs as alternatives to warfarin for secondary prevention of recurrent as well as primary prevention of first stroke in patients with $\mathrm{AF}$ [29].

\section{RENAL IMPAIRMENT}

Patients with AF and renal dysfunction are at increased risk of both ischemic and bleeding events [22-24]. Warfarin treatment reduces the risk of stroke or systemic embolism in patients with chronic kidney disease, but warfarin and aspirin are associated with increased risks of bleeding. In the RE-LY trial, the risk of major bleeding with dabigatran or warfarin was a $>2$-fold higher in patients with a $\mathrm{Cr}_{\mathrm{Cl}}<50 \mathrm{~mL} / \mathrm{min}$ compared with those with clearance $\geq 80 \mathrm{~mL} / \mathrm{min}$., but the relative increase in bleeding risk was similar for both drugs [25] Dabigatran is approximately $80 \%$ excreted via the renal route, and higher concentrations of the drug accumulate in the blood of patients with renal dysfunction [26]. Dabigatran is contraindicated in patients with estimated $\mathrm{Cr}_{\mathrm{Cl}}<30 \mathrm{~mL} / \mathrm{min}$ in Europe and Canada, and the $75 \mathrm{mg}$ b.i.d. dose is approved for use in patients with $\mathrm{Cr}_{\mathrm{Cl}} 15-29 \mathrm{ml} / \mathrm{min}$ in the US.

In ROCKET AF, $20.7 \%$ of the trial cohort had moderate renal impairment $\left(\mathrm{Cr}_{\mathrm{Cl}} 30-49 \mathrm{~mL} / \mathrm{min}\right)$. Patients with moderate renal impairment were given a reduced dose of rivaroxaban (15 mg once daily). Results of a pre-specified secondary analysis of patients with renal impairment were consistent with the overall trial results [27]. Among those with $\mathrm{Cr}_{\mathrm{Cl}} 30$ $49 \mathrm{~mL} / \mathrm{min}$, the primary endpoint of stroke or systemic embolism occurred in 2.32 per 100 patient-years with rivaroxaban $15 \mathrm{mg} /$ day vs. 2.77 per 100 patient-years with warfarin (HR $0.84 ; 95 \%$ CI $0.57-1.23$ ) in the per-protocol population. Intention-to-treat analysis yielded similar results (HR 0.86 ; $95 \%$ CI $0.63-1.17)$. Rates of major and clinically relevant non-major bleeding (17.82 vs. $18.28 / 100$ patient-years; $\mathrm{p}=0.76)$ and hemorrhagic stroke ( 0.71 vs. $0.88 / 100$ patientyears, $\mathrm{p}=0.54$ ) were similar with rivaroxaban or warfarin. Fatal bleeding ( 0.28 vs. $0.74 / 100$ patient-years, $p=0.047)$ occurred less often with rivaroxaban. Since clinical data are limited, rivaroxaban should be used with caution in patients with severe renal impairment $\left(\mathrm{Cr}_{\mathrm{Cl}}<30 \mathrm{ml} / \mathrm{min}\right.$ and in those with renal impairment concomitantly receiving other drugs that increase the plasma concentration of rivaroxaban. Similarly to observations with rivaroxaban and dabigatran, the subgroup of patients with renal impairment in the ARISTOTLE trial tracked the main study results; the hazard ratio for bleeding was even lower when the GFR was low [28]. Although the U.S. FDA has allowed labeling of apixaban for patients with end-stage renal disease receiving hemodialysis, clinical data on safety and efficacy in this population are lacking.

\section{PATIENT AGE}

The risk of bleeding among patients randomized in the RE-LY trial increased with age, and compared with warfarin both doses of dabigatran were associated with an increasing relative risk of major bleeding with increasing age categories $(<65,65$ to $74, \geq 75$ years; interaction $\mathrm{p}<0.001$ for each analysis) [25]. Compared with warfarin, dabigatran $110 \mathrm{mg}$ b.i.d. was associated with a lower risk of major bleeding ( 2.87 vs $3.57 \%$; $p=0.002$ ), whereas dabigatran $150 \mathrm{mg}$ b.i.d. was associated with a similar risk of major bleeding (3.31 vs $3.57 \% ; p=0.32$ ). There was a significant treatment-by-age interaction, such that dabigatran $110 \mathrm{mg}$ b.i.d. was associated with a lower risk of major bleeding in patients aged $<75$ years old $(1.89 \%$ vs $3.04 \% ; P<0.001)$ and a similar risk in those $\geq 75$ years old $(4.43$ versus $4.37 \%$; $p=0.89$; interaction $\mathrm{p}<0.001$ ), whereas dabigatran $150 \mathrm{mg}$ b.i.d. was associated with a lower risk of major bleeding in those aged $<75$ years (2.12 vs $3.04 \% ; p<0.001)$ and a trend toward higher risk of major bleeding in those $\geq 75$ years old $(5.10$ vs $4.37 \%$; 
$p=0.07$; interaction $\mathrm{p}<0.001)$. The interaction with age was evident for extracranial bleeding, but not for intracranial bleeding, with the risk of the latter consistently reduced with dabigatran compared with warfarin in patients of all ages. There was no significant interaction between treatment and $\mathrm{Cr}_{\mathrm{Cl}}$ for major bleeding, suggesting that other age-related factors are more important than renal function in determining bleeding risk in elderly patients treated with dabigatran. Higher blood concentrations of dabigatran with increasing age might contribute to the higher risk of extracranial bleeding with dabigatran compared with warfarin in elderly patients.

Data regarding age-related bleeding in the ROCKET AF trial of rivaroxaban are available only in preliminary form [30]. Compared to younger patients, those $\geq 75$ years old at entry had a mean $\mathrm{CHADS}_{2}$ score of 3.7 vs. 3.3, and a higher proportion were female (46 vs. 35\%). During 9,247 patientyears exposure, 429 stroke or systemic embolic events occurred among patients $\geq 75$ years old. The on-treatment thromboembolism rates of 1.78 vs. 2.65 per 100 patientyears with rivaroxaban and warfarin, respectively (HR 0.67 ; 95\% CI 0.51-0.89) were not much higher than in younger patients. By intention-to-treat analysis, rates of stroke and systemic embolism were 2.29 vs 2.85 per 100 patient-years among older patients treated with rivaroxaban vs warfarin, respectively (HR 0.80 ; 95\% CI 0.63-1.02), compared with 2.00 vs 2.10 per 100 patient-years among those under age 75 years (HR 0.95 ; 95\% CI 0.76-1.19; interaction $p=0.3131$ for differences in treatment effect related to age). Older patients had higher rates of (mostly non-major) clinically relevant bleeding (interaction $p=0.009$ ), but rates with warfarin and rivaroxaban were comparable. Hence, among the relatively high-risk elderly patients enrolled in ROCKET AF, rivaroxaban performed favorably compared to adjusted-dose warfarin, as it did in the overall ROCKET-AF population. A subgroup analysis of the response of elderly patients to apixaban in the ARISTOTLE trial found greater absolute benefit of apixaban in the elderly due to the higher risk associated with age. Apixaban was more effective than warfarin in preventing stroke, reducing mortality and associated with less major bleeding, total bleeding, and intracranial hemorrhage regardless of age (interaction $\mathrm{p}>0.11$ for all analyses) [30a].

\section{OTHER PERTINENT CLINICAL SUBGROUPS}

Subgroup analyses of other clinically important subgroups of patients with AF in trials comparing the NOACs with warfarin have been reported, including those stratified by center-based quality of warfarin anticoagulation control (time in therapeutic range [TTR]). In the RE-LY trial, the benefits of dabigatran, $150 \mathrm{mg}$ b.i.d. at reducing stroke, the $110 \mathrm{mg}$ b.i.d. dose at reducing bleeding, and both doses at reducing hemorrhagic stroke were consistent irrespective of the quality of INR control, though in terms of all ischemic events and mortality, the advantages of dabigatran were greater at sites with poor INR control than at those with good INR control [31]. Since the quality of anticoagulation management generally parallels other aspects of health care quality, rates of adverse clinical events, including stroke and bleeding are typically lower at clinical centers where higher TTR values are achieved. While patients receiving wellcontrolled warfarin therapy may gain less advantage from substitution of one of the NOACs in terms of efficacy, the subgroup analyses are typically under-powered to support firm conclusions, and there is little evidence that the lower rates of hemorrhagic stroke during NOAC therapy are related to the TTR of the warfarin comparator [31a].

Studies of other subgroups, including patients classified as naïve to warfarin prior to randomization, those with diabetes mellitus or heart failure, and those delineated by ethnicity or geographical region, have generally been consistent with the main trial results (interaction $p$-values for these variables not significant). These secondary analyses must be considered exploratory, however, and additional studies will be needed in independently selected cohorts, to identify specific patient characteristics associated with greater or lesser efficacy or efficacy of the NOACs when used for stroke prevention.

\section{CONCLUSIONS}

The NOACs studied in phase III clinical trials completed to date have displayed generally favorable efficacy and safety profiles compared to warfarin. For patients on stable, long-term doses of warfarin, the advantage of substituting one of these new agents is less well established, and additional data on cost-effectiveness and safety during longer periods of exposure may inform prescribing practice. In patients with poorly controlled INR values in follow-up, or with new therapy initiation, the potential advantages of the NOACS are likely to be most manifest. Although comparisons of one NOAC another is complicated by differences in patient population, study design and other methodological factors [32], all represent viable alternatives to warfarin for stroke prevention in patients with nonvalvular AF.

\section{DISCLOSURES}

Dr. Dorian has received consulting fees from the following companies involved in developing and/or marketing of anticoagulant drugs for prevention of thromboembolism: Bayer HealthCare, Boehringer Ingelheim, Bristol - Myers Squibb, and Pfizer.

\section{CONFLICT OF INTEREST}

The authors confirm that this article content has no conflict of interest.

\section{ACKNOWLEDGEMENTS}

Declared none.

\section{REFERENCES}

[1] Independent predictors of stroke in patients with atrial fibrillation. Neurology 2007; 69: 546-4.

[2] Wang TJ, Massaro JM, Levy D, et al. A risk score for predicting stroke or death in individuals with new-onset atrial fibrillation in the community: the Framingham Heart Study. JAMA 2003; 290: 1049-56.

[3] Hart RG, Pearce LA, Aguilar MI. Meta-analysis: antithrombotic therapy to prevent stroke in patients who have nonvalvular atrial fibrillation. Ann Intern Med 2007; 146: 857-67.

[4] Diener HC. Stroke prevention using the oral direct thrombin inhibitor ximelagatran in patients with non-valvular atrial fibrillation: Pooled analysis from the SPORTIF III and V studies. Cerebrovasc Dis 2006; 21: 279-93. 
[5] Connolly S, Ezekowitz M. Correspondence: Dabigatran versus warfarin in patients with atrial fibrillation. N Engl J Med 2009; 361: 2671-5

[6] Connolly SJ, Ezekowitz MD, Yusuf S, et al. Dabigatran versus warfarin in patients with atrial fibrillation. N Engl J Med 2010; 363: $1875-6$.

[7] Hariharan S, Madabushi R. Clinical pharmacology basis of deriving dosing recommendations for dabigatran in patients with severe renal impairment. J Clin Pharmacol 2012; 52 (suppl): 119S25S.

[8] Patel MR, Mahaffey KW, Garg J, et al. Rivaroxaban versus warfarin in nonvalvular atrial fibrillation. N Engl J Med 2011; 365(10): 883-91.

[9] Granger CB, Alexander JH, McMurray JJV, et al. Apixaban versus warfarin in patients with atrial fibrillation. N Engl J Med 2011; 365: 981-92.

[10] Connolly SJ, Eikelboom J, Joyner C, et al. Apixaban in patients with atrial fibrillation. N Engl J Med 2011; 364: 806-17.

[11] Ruff CT, Giugliano RP, Antman EM, et al. Evaluation of the novel factor Xa inhibitor edoxaban compared with warfarin in patients with atrial fibrillation: Design and rationale for the Effective aNticoaGulation with factor $\mathrm{xA}$ next GEneration in Atrial Fibrillation-Thrombolysis In Myocardial Infarction study 48 (ENGAGE AF-TIMI 48). Am Heart J 2010; 160: 635-41.

[12] Hohnloser SH, Oldgren J, Yang S, et al. Myocardial Ischemic Events in Patients With Atrial Fibrillation Treated With Dabigatran or Warfarin in the RE-LY (Randomized Evaluation of Long-Term Anticoagulation Therapy) Trial / Clinical Perspective. Circulation. 2012; 125: 669-76.

[12a] Food and Drug Administration. FDA study of Medicare patients finds risks lower for stroke and death but higher for gastrointestinal bleeding with Pradaxa (dabigatran) compared to warfarin [press release]. May 13, 2014 (http://www.fda.gov/Drugs/DrugSafety/ ucm396470.htm)

[13] Uchino K, Hernandez AV. Dabigatran association with higher risk of acute coronary events: meta-analysis of noninferiority randomized controlled trials. Arch Intern Med 2012; 172: 397-402.

[14] Mega JL, Braunwald E, Wiviott SD, et al. Rivaroxaban in patients with a recent acute coronary syndrome. N Engl J Med. 2012; 366: 9-19.

[15] APPRAISE Steering Committee and Investigators. Apixaban, an oral, direct, selective factor Xa inhibitor, in combination with antiplatelet therapy after acute coronary syndrome: Results of the Apixaban for Prevention of Acute Ischemic and Safety Events (APPRAISE) Trial. Circulation 2009; 119: 2877-85.

[16] Hart RG, Diener HC, Yang S, et al. Intracranial hemorrhage in atrial fibrillation patients during anticoagulation with warfarin or dabigatran: The RE-LY trial. Stroke 2012; 43: 1511-7.

[17] Stroke in Atrial Fibrillation Working Group. Independent predictors of stroke in atrial fibrillation: A systematic review. Neurology 2007; 69: 546-54.

[18] Palareti G, Cosmi B. Bleeding with anticoagulation therapy: Who is at risk, and how best to identify such patients. Thromb Haemost 2009; 102: 268-78.

[19] Diener H-C, Connolly SJ, Ezekowitz MD, et al. Dabigatran compared with warfarin in patients with atrial fi brillation and previous transient ischaemic attack or stroke: a subgroup analysis of the RE-LY trial. Lancet Neurol 2010; 9: 1157-63.

[20] Hankey G, Patel M, Stevens S, et al. Rivaroxaban compared with warfarin in patients with atrial fibrillation and previous stroke or transient ischaemic attack: a subgroup analysis of ROCKET AF. Lancet Neurol 2012; 11: 315-22.
[21] Easton JD, Lopes RD, Bahit MC, et al. Apixaban compared with warfarin in patients with atrial fibrillation and previous stroke or transient ischaemic attack: a subgroup analysis of the ARISTOTLE trial. Lancet Neurol 2012; 11: 503-11.

[22] Go AS, Fang MC, Udaltsova N, et al. Impact of proteinuria and glomerular filtration rate on risk of thromboembolism in atrial fibrillation: the anticoagulation and risk factors in atrial fibrillation (ATRIA) study. Circulation 2009; 119: 1363-9.

[23] Oleson JB, Lip GHY, Kamper A-L, et al. Stroke and bleeding in atrial fibrillation with chronic kidney disease. N Engl J Med 2012; 367: 625-35.

[24] Piccini JP, Stevens SR, Chang YC, et al. Renal dysfunction as a predictor of stroke and systemic embolism in patients with nonvalvular atrial fibrillation: Derivation and validation of the $\mathrm{R}_{2} \mathrm{CHADS}_{2}$ index in the ROCKET AF and ATRIA study cohorts. Circulation 2013; 127(2): 224-32.

[25] Eikelboom JW, Wallentin L, Connolly SJ, et al. Risk of bleeding with 2 doses of dabigatran compared with warfarin in older and younger patients with atrial fibrillation: An analysis of the Randomized Evaluation of Long-term Anticoagulant Therapy (RELY) trial. Circulation 2011; 123: 2363-72.

[26] Eriksson BI, Quinlan DJ, Weitz JI. Comparative pharmacodynamics and pharmacokinetics of oral direct thrombin and factor Xa inhibitors in development. Clin Pharmacokinet 2009; 48: 1-22.

[27] Fox KAA, Piccini JP, Wojdyla D, et al. Prevention of stroke and systemic embolism with rivaroxaban compared with warfarin in patients with non-valvular atrial fibrillation and moderate renal impairment. Eur Heart J 2011; 32(19): 2387-94.

[28. Hohnloser SH, Hijazi Z, Thomas L, Alexander JH, et al. Efficacy of apixaban when compared with warfarin in relation to renal function in patients with atrial fibrillation: insights from the ARISTOTLE trial. Eur Heart J 2012; 33: 2821-30.

[29] Ntaios G, Papavasileiou V, Diener HC, et al. Nonvitamin-kantagonist oral anticoagulants in patients with atrial fibrillation and previous stroke or transient ischemic attack: A systematic review and meta-analysis of randomized controlled trials. Stroke 2012; 43: 3298-304.

[30] Halperin JL, Wojdyla D, Piccini JP, et al. Efficacy and safety of rivaroxaban compared with warfarin among elderly patients with nonvalvular atrial fibrillation in the ROCKET-AF trial. Stroke 2012; 43 Suppl, A-148.

[30a] Halvorsen S, Atar D, Yang H, et al. Efficacy and safety of apixaban compared with warfarin according to age for stroke prevention in atrial fibrillation: observations from the ARISTOTLE trial. Eur Heart J 2014; Feb 20. [Epub ahead of print]

[31] Wallentin L, Yusuf S, Ezekowitz MD, et al. Effi cacy and safety of dabigatran compared with warfarin at different levels of international normalised ratio control for stroke prevention in atrial fibrillation: An analysis of the RE-LY trial. Lancet 2010; 376: 975-83.

[31a] Piccini JP, Hellkamp AS, Lokhnygina Y, et al. Relationship between time in therapeutic range and comparative treatment effect of rivaroxaban and warfarin: results from the ROCKET AF trial. $\mathrm{J}$ Am Heart Assoc 2014; (Published online April 22, 2014; 3:e000521. doi: 10.1161/JAHA.113.000521.)

[32] Spyropoulos AC, Goldenberg NA, Kessler CM, et al. Comparative effectiveness and safety of the novel oral anticoagulants: Do the pivotal clinical trials point to a new paradigm? J Thromb Haemost 2012; 10: 2621-4. 